\title{
Kasuistik: Management eines limbalen Carcinoma in situ der Bindehaut
}

\author{
Max Brinkmann · Salvatore Grisanti · Mariella Fleischer · Armin Mohi
}

Eingegangen: 12. März 2020 / Angenommen: 21. November 2020 / Online publiziert: 21. Dezember 2020

(c) Der/die Autor(en) 2020

\begin{abstract}
Zusammenfassung Das Carcinoma in situ (CIS) der Bindehaut stellt einen der häufigsten okulären Tumoren dar. Neben der klassischen chirurgischen Therapie besteht die Möglichkeit der Anwendung von Chemotherapeutika adjuvant oder als Monotherapie. Wir behandelten einen Patienten mit einem limbalen CIS ausschließlich konservativ mittels Applikation von Mitomycin(MMC)-Augentropfen. Drei Monate nach Abschluss zeigte sich kein Hinweis auf Dysplasien oder Malignität, und auch nach 12 Monaten ergab sich kein klinischer Hinweis auf ein Rezidiv. Bei der Therapie steht zwar weiterhin die chirurgische Sanierung im Vordergrund, in den letzten 15 Jahren zeigt sich jedoch ein Trend zu topischen Chemotherapeutika primär oder adjuvant bei einer Resektion nicht im Gesunden. Das Nebenwirkungspotenzial sowie der infrastrukturelle Aufwand sollten dabei stets berücksichtigt werden.
\end{abstract}

Schlüsselwörter Intraepitheliales Karzinom . Präinvasives Karzinom · Intraepitheliale Neoplasmen · Okuläre Chemotherapie · Mitomycin Augentropfen
Case report: Management of a Carcinoma in situ (CIS) of the limbal conjunctiva

Summary The carcinoma in situ (CIS) of the conjunctiva is one of the most common forms of ocular surface squamous neoplasia (OSSN). Besides classical surgical excision, treatment options involve application of adjuvant chemotherapeutics or as monotherapy. We treated a patient with limbal CIS exclusively conservatively by applying mitomycin C (MMC) eyedrops. There were no signs of dysplasia or malignancy 3 months after the treatment and even after 12 months there were no clinical findings of recurrence. Surgical removal remains the primary treatment for OSSN. Nevertheless, for the past 15 years there has been a trend to consider adjuvant or primary topical chemotherapy as a treatment option, especially if removal of the lesion was incomplete. The potential side effects and the extensive infrastructural requirements should always be taken into consideration.

Keywords Carcinoma, intraepithelial · Carcinoma, preinvasive $\cdot$ Neoplasms, intraepithelial $\cdot$ Ocular chemotherapy $\cdot$ Mitomycin eyedrops

\section{Einleitung}

\section{Anamnese}

Uns vorstellig wurde ein 82-jähriger Patient mit anhaltendem Fremdkörpergefühl am linken Auge. Die seitens des vorbehandelnden Arztes ordinierte Therapie mit Tränenersatzmittel beidseits sowie am linken Auge VitA-POS-Augensalbe (Ursapharm, Saarbrücken, Deutschland) zur Nacht zeigte keine Besserung. Neben Fremdkörpergefühl klagte der Patient über keine weiteren Symptome. 

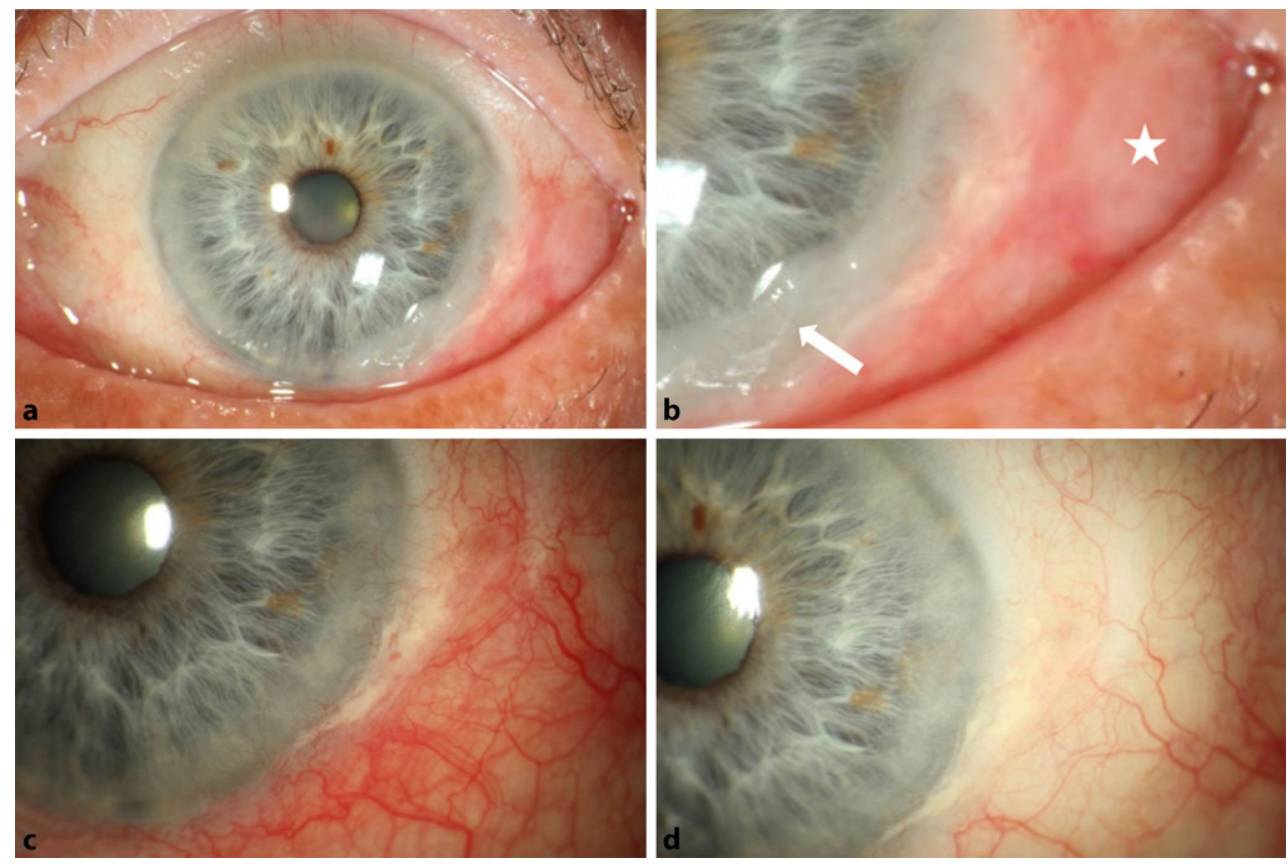

Abb. 1 Der Ursprungsbefund $(\mathbf{a}, \mathbf{b})$ zeigt temporal eine deutlich prominente Bindehautläsion mit gelatinöser Infiltration bis in die Hornhaut mit begleitender Hornhautvaskularisation (Pfeil). Im Bindehautbereich imponierte zudem eine beginnende Verhornung des Epithels (Stern). Nach 4 Zyklen MMC-Therapie (c) zeigte sich neben einer konjunktivalen Injektion ein

deutlicher Rückgang der Bindehautläsion. Auch die Hornhautinfiltration war zudem rückläufig. Nach 6 Zyklen MMC-Therapie erscheint ein vollständiger Rückgang der Bindehautprominenz (d). Eine Infiltration der Hornhaut ließ sich nicht mehr beobachten, und auch die konjunktivale Injektion zeigte sich deutlich vermindert

\section{Klinischer Befund}

Klinisch zeigte sich am linken Auge temporal eine deutlich prominente Bindehautläsion mit gelatinöser Infiltration bis in die Hornhaut mit begleitender Hornhautvaskularisation. Im Bindehautbereich imponierte zudem eine beginnende Verhornung des Epithels. Der Gesamtbefund war zur Unterlage kaum verschieblich (Abb. 1).

\section{Diagnose}

Vom klinischen Befund her stellte sich der Verdacht auf einen malignen Bindehautprozess. Daher führten wir eine Biopsie im Bindehautbereich durch, um eine histologische Begutachtung durchführen zu lassen. Dabei zeigte sich hyperplastisches Epithel mit atypischen Zellen und pleomorphen Zellkernen in allen Epithellagen sowie einer deutlichen Schichtungsstörung. Die Basalmembran wurde respektiert (Abb. 2). Außerdem fanden sich Dyskeratosen und Nekrosen sowie eine beginnende Verhornung im Epithel und um die Becherzellen. Somit ließ sich die Diagnose eines Carcinoma in situ (CIS) stellen.

\section{Therapie und Verlauf}

Nachdem die Diagnose eines CIS histologisch gesichert war, stellte sich die Frage nach der Therapie. Eine chirurgische Totalexzision stellt in diesem Bereich

eine Herausforderung dar. Der Bindehautlimbusbefall sowie das hohe Patientenalter mussten dabei berücksichtigt werden, da die Limbusstammzellinsuffizienz eine der bekannten Komplikationen der chirurgischen Therapie darstellt.

Gemeinsam mit dem Patienten entschieden wir uns für ein konservatives Vorgehen mit der Anwendung von Mitomycin(MMC)-Augentropfen (Apotheke des Universitätsklinikums Schleswig-Holstein, Lübeck, Deutschland) in einer Konzentration von 0,04\%, da dies bereits seit vielen Jahren in der Behandlung von okulären Tumoren Anwendung findet und eine entsprechend große Anwendungserfahrung mit guten Erfolgsquoten in der Literatur beschrieben worden ist [7].

Die Therapie erfolgte zunächst in 4 Zyklen. Jeder Zyklus umfasste 7 Tage, während dieser die MMC-Augentropfen 4-mal pro Tag (alle $6 \mathrm{~h}$ ) appliziert wurden. Zudem wurden die Tränenpünktchen okkludiert, um eine systemische Aufnahme zu reduzieren. $\mathrm{Zu}$ sätzlich erfolgte eine intensive Tränenersatzmitteltherapie. Aufgrund mangelnder Compliance erfolgten die Anwendungszyklen im stationären Setting. Auf jeden Zyklus folgte eine 1-wöchige Pause mit intensiver Tränenersatzmitteltherapie.

Nach Abschluss der 4 Zyklen führten wir nach 12 Wochen eine Reevaluation und erneute klinische Begutachtung inklusive Impressionszytologie im Bereich des Limbus sowie der Bindehaut durch. Klinisch zeigte sich zu diesem Zeitpunkt neben einer kon- 


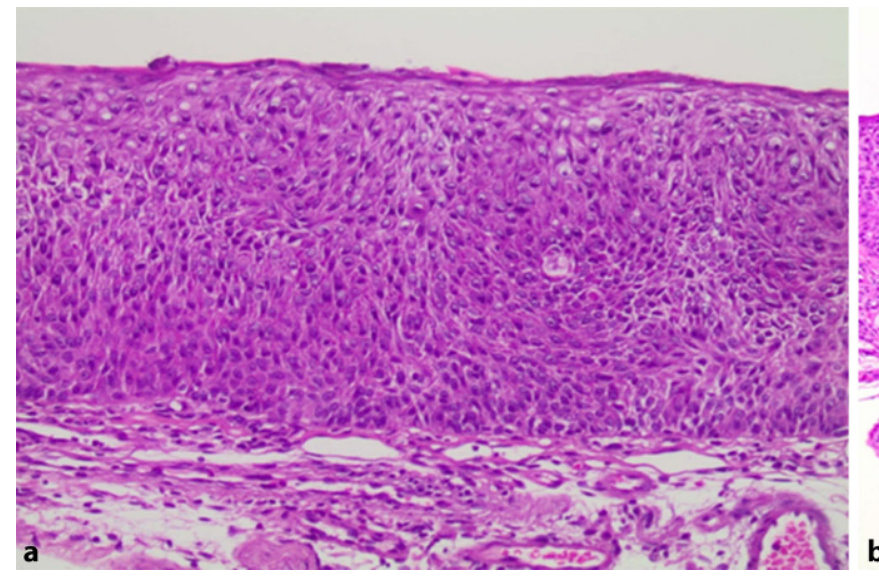

Abb. 2 Histologiebefund der Biopsie, welche vor Behandlungsbeginn durchgeführt wurde (a Detailaufnahme, b Übersichtsaufnahme). Es zeigt sich hyperplastisches Epithel mit atypischen Zellen und pleomorphen Zellkernen in allen Epi-

junktivalen Injektion ein deutlicher Rückgang der Bindehautläsion. Insbesondere die Hornhautinfiltration zeigte sich rückläufig (Abb. 1). In der Impressionszytologie fanden sich im Bereich der Bindehaut noch vereinzelte atypische Zellen, die für Zellen einer neoplastischen Dysplasie sprachen.

Aufgrund des Befundes entschieden wir uns dazu, 2 weitere Zyklen der MMC-Therapie (nach oben genanntem Schema) durchzuführen. Anschließend erfolgte erneut eine klinische und histologische Kontrolle.

Nach insgesamt 6 Zyklen MMC-Therapie zeigte sich ein vollständiger Rückgang der Bindehautprominenz. Eine Infiltration der Hornhaut ließ sich nicht mehr beobachten, und auch die konjunktivale Injektion zeigte deutlich vermindert (Abb. 1).

Die impressionszytologische Untersuchung ergab keinen Nachweis von Dysplasien und insbesondere keinen Anhalt für Malignität.

Drei Monate nach Abschluss des letzten Zyklus erfolgte eine erneute impressionszytologische Begutachtung, bei der kein Hinweis auf Dysplasien oder Malignität mehr nachgewiesen werden konnte. Weitere Kontrollen fanden im Abstand von 12 Wochenintervallen statt und ergaben auch nach einem Nachbeobachtungszeitraum von 12 Monaten einen stabilen klinischen Befund ohne Hinweise auf ein Rezidiv weder klinisch noch histopathologisch.

Der Patient ist aktuell weiterhin beschwerdefrei.

\section{Diskussion}

Das Carcinoma in situ der Bindehaut gehört zu der Gruppe der sog. Ocular Surface Squamous Neoplasia (OSSN). Die durchschnittliche Inzidenz beträgt 0,18/ Jahr/100.000 für Männer und 0,08/Jahr/100.000 für Frauen. Damit stellen OSSN die dritthäufigsten okulären Tumoren überhaupt dar, wobei erhebliche geografische Schwankungen mit dem Hauptauftreten in

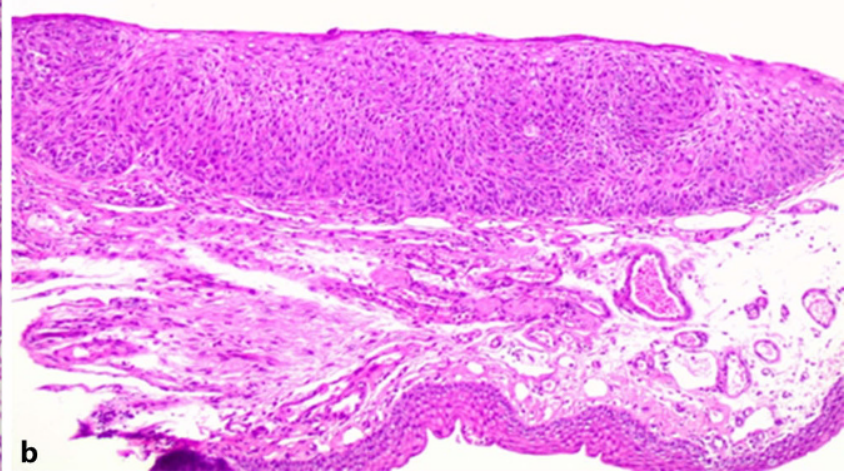

thellagen sowie einer deutlichen Schichtungsstörung, die Basalmembran wird respektiert. Außerdem fanden sich Dyskeratosen und Nekrosen, sowie eine beginnende Verhornung im Epithel und um die Becherzellen

Afrika zu beobachten sind [3]. Als Hauptrisikofaktoren werden UV-Licht-Exposition sowie eine Infektion mit dem HI-Virus betrachtet. Weitere Risikofaktoren sind Nikotinabusus, Kontakt mit Petroleumprodukten sowie Alter [5].

Traditionell besteht die Therapie in der alleinigen chirurgischen Exzision mit Kryotherapie der Wundränder [1, 10]. Für dieses therapeutische Verfahren stellten Peksaya et al. in einer retrospektiven Studie Erfolgsraten von bis zu 89,3\% bei Patienten mit konjunktivaler intraepithelialer Neoplasie (CIN) fest [8]. In derselben Studie betrug der Anteil der Patienten ohne Rezidiv nach 1 Jahr 91\% [8]. Oftmals erstreckt sich jedoch das mikroskopische Ausmaß der Erkrankung über die Grenzen des klinisch sichtbaren Befundes hinaus [6]. So ergeben sich in der Literatur Rezidivraten von bis zu $56 \%$ bei der alleinigen chirurgischen Exzision. Selbst bei Behandlungen, bei denen postoperativ tumorfreie Schnittränder in der histopathologischen Begutachtung festgestellt wurden, liegt die Wahrscheinlichkeit eines Wiederauftretens immer noch bei $33 \%$ [11].

Chemotherapeutika, sowohl adjuvant als auch als Monotherapie genutzt, haben in den letzten Jahren erheblich an Popularität gewonnen [1]. Aktuell werden verschiedene topische Medikamente adjuvant zur chirurgischen Exzision verwendet $[4,8,9]$. Bei diesem Vorgehen konnten für alle Entitäten der OSSN gute Erfolgsraten erzielt werden [9]. Die Chemotherapeuti$\mathrm{ka}$, die dabei benutzt werden, umfassen Mitomycin-C, 5 -Fluorouracil (5-FU) und Interferon- $\alpha$-2b (IFN $\alpha 2 b$ ) [6].

IFNa2b kann subkonjunktival oder topisch als Augentropfen in einer Konzentration von $1 \mathrm{Mio}$. IE/ml angewandt werden. Dabei konnte in retrospektiven Analysen für die Behandlung der CIN eine Erfolgsrate von etwa $80 \%$ beobachtet werden [2]. IFN $\alpha 2 b$ gibt es nicht als Fertigpräparat, sodass es von spezialisierten 
Apotheken hergestellt werden muss, was die Anwendung häufig erschwert.

MMC-Augentropfen stellen ebenfalls eine effektive Therapieoption für OSSN dar. Dabei liegen die Erfolgsraten bei primärer Anwendung zwischen 80 und $100 \%$ für keratokonjunktivale CIS [7]. In der klinischen Anwendung werden v. a. die Konzentration $0,02 \%$ oder $0,04 \%$ verwendet, wobei für die niedrigere Konzentration die Möglichkeit einer durchgängigen Anwendung über 1 Monat beschrieben wird. Die höhere Konzentration wird üblicherweise in 1-wöchigen Zyklen verabreicht [7]. Als Nachteile sind Augenschmerzen, Tränenwegstenosen, limbale Stammzellschäden sowie toxische Effekte an der Augenoberfläche beschrieben [6]. Bei der Anwendung sollte außerdem eine zeitweise Okklusion der Tränenpünktchen erfolgen, um systemische Nebenwirkungen zu vermeiden.

Die Anwendung von 5-FU (1\%) erfolgt 4-mal pro Tag für 1 Monat und einer anschließenden Pause für 3 Monate. Dabei zeigten sich Erfolgsraten von bis zu $100 \%$ [7]. Die Rezidivraten liegen zwischen 4,5 und $20 \%[4,12]$. Als nachteilig sind Augenreizung und gelegentliche Konjunktivitiden beschrieben.

Prinzipiell sind alle der hier beschriebenen Chemotherapeutika für die Behandlung der OSSN geeignet. Vergleichende Studien ergaben schwankende Ergebnisse die Rezidiv- und Erfolgsquoten betreffend. Eine ambulante Therapie ist grundsätzlich möglich. Hierbei muss jedoch berücksichtigt werden, dass es die genannten Chemotherapeutika als Augentropfen nicht als Fertigpräparat zu kaufen gibt. Die Herstellung erfolgt in der Regel in spezialisierten Apotheken. Entsprechend dem Nebenwirkungspotenzial muss zudem auf eine gute Patientencompliance geachtet werden.

Bei der Therapieplanung muss neben verschiedenen anderen Aspekten auch insbesondere auf die Lage der Läsion geachtet werden, da diese einen großen Einfluss auf das Auftreten von Komplikationen wie Symblepharonbildung, Wundheilungsstörung und Stammzellinsuffizienz mit konsekutiver Hornhautvaskularisation hat. In dem vorliegenden Fall wären bei einer chirurgischen Therapie eine Keratektomie im Limbusbereich sowie Nachresektion der Bindehaut und anschließende Rekonstruktion notwendig gewesen. Wir entschieden uns deshalb für ein konservatives Vorgehen. Die in der Literatur beschriebene hohe Erfolgsquote sowie die Anwendung in Zyklen bewegten uns schließlich, MMC-Augentropfen im stationären Rahmen als Monotherapie zu verwenden. Im hier beschriebenen Fall konnte damit bei einem bisherigen Nachbeobachtungszeitraum von 12 Monaten ein guter rezidivfreier Erfolg erzielt werden.

Bei der Abwägung der verschiedenen Therapieformen sollte unbedingt beachtet werden, dass ein rein konservatives Vorgehen nur für Subgruppen (CIS und CIN) der Bindehauttumoren Erfolg versprechend ist. Eine Inzisionsbiopsie kann selbstverständlich immer nur einen Teilbereich der Läsion abbilden. Somit können weitere Veränderungen wie das Vorliegen eines Plattenepithelkarzinoms nur bedingt ausgeschlossen werden. Weiterhin ist zu berücksichtigen, dass eine Impressionszytologie, die auch in diesem Kasus Anwendung fand, keine Auskunft über die Tiefenausdehnung des Befundes geben kann.

Zusammenfassend ist festzuhalten, dass neben der klassischen chirurgischen Therapie auch etablierte konservative Therapieoptionen existieren. Die beschriebenen Optionen der topischen Anwendung von verschiedenen Chemotherapeutika als adjuvante [4, 8, 9] oder alleinige Therapie stellen bei einigen Subgruppen der OSSN, insbesondere bei den CIS und CIN, eine gute Alternative dar.

Funding Open Access funding enabled and organized by Projekt DEAL.

Interessenkonflikt M. Brinkmann, S. Grisanti, M. Fleischer und A. Mohi geben an, dass kein Interessenkonflikt besteht.

Ethics approval The study was approved by the local ethics committee. All study components complied with the Health Insurance Portability and Accountability Act of 1996 and adhered to the tenets of the Declaration of Helsinki.

Open Access Dieser Artikel wird unter der Creative Commons Namensnennung 4.0 International Lizenz veröffentlicht, welche die Nutzung, Vervielfältigung, Bearbeitung, Verbreitung und Wiedergabe in jeglichem Medium und Format erlaubt, sofern Sie den/die ursprünglichen Autor(en) und die Quelle ordnungsgemäß nennen, einen Link zur Creative Commons Lizenz beifügen und angeben, ob Änderungen vorgenommen wurden.

Die in diesem Artikel enthaltenen Bilder und sonstiges Drittmaterial unterliegen ebenfalls der genannten Creative Commons Lizenz, sofern sich aus der Abbildungslegende nichts anderes ergibt. Sofern das betreffende Material nicht unter der genannten Creative Commons Lizenz steht und die betreffende Handlung nicht nach gesetzlichen Vorschriften erlaubt ist, ist für die oben aufgeführten Weiterverwendungen des Materials die Einwilligung des jeweiligen Rechteinhabers einzuholen.

Weitere Details zur Lizenz entnehmen Sie bitte der Lizenzinformation auf http://creativecommons.org/licenses/by/4. 0/deed.de.

\section{Literatur}

1. Adler E, Turner JR, Stone DU. Ocular surface squamous neoplasia: a survey of changes in the standard of care from 2003 to 2012. Cornea. 2013;12:1558-61.

2. Galor A, Karp CL, Chhabra S, et al. Topical interferon alpha $2 \mathrm{~b}$ eye-drops for treatment of ocular surface squamous neoplasia: a dose comparison study. Br J Ophthalmol. 2010;94:551-4.

3. Gichuhi S, Ohnuma S, Sagoo MS, Burton MJ. Pathophysiology of ocular surface squamous neoplasia. Exp Eye Res. 2014;129:172-82.

4. Midena E, Angeli CD, Valenti M, et al. Treatment of conjunctival squamous cell carcinoma with topical 5-fluorouracil. BrJOphthalmol.2000;84:268-72. 
5. Moon CS, Nanji AA, Galor A, McCollister KE, Karp CL. Surgical versus medical treatment of ocular surface squamous neoplasia: a cost comparison. Ophthalmology. 2016;123(3):497-504.

6. Nanji AA, Moon CS, Galor A, Sein J, Oellers P, Karp CL. Surgical versus medical treatment of ocular surface squamous neoplasia: a comparison of recurrences and complications. Ophthalmology. 2014;121(5):994-1000.

7. Nanji AA, Sayyad FE, Karp CL. Topical chemotherapy for ocular surface squamous neoplasia. Curr Opin Ophthalmol.2013;24(4):336-42.

8. Peksayar G, Altan-Yaycioglu R, Onal S. Excision and cryosurgery in the treatment of conjunctival malignant epithelial tumours. Eye (Lond). 2003;17:228-32.

9. Siganos CS, Kozobolis VP, Christodoulakis EV. The intraoperative use of mitomycin-C in excision of ocular surface neoplasia with or without limbal autograft transplantation. Cornea. 2002;21:12-6.

10. Stone DU, Butt AL, Chodosh J. Ocular surface squamous neoplasia: a standard of care survey. Cornea. 2005;24:297-300.

11. Tabin G, Levin S, Snibson G, et al. Late recurrences and the necessity for long-term follow-up in corneal and conjunctivalintraepithelialneoplasia. Ophthalmology. 1997;104:485-92.

12. Venkateswaran N, Mercado C, Galor A, Karp C. Comparison of topical 5-fluorouracil and interferon alfa-2b as primary treatment modalities for ocular surface squamous neoplasia. Am J Ophthalmol.2019;199:216-22.

Hinweis des Verlags Der Verlag bleibt in Hinblick auf geografische Zuordnungen und Gebietsbezeichnungen in veröffentlichten Karten und Institutsadressen neutral. 